\title{
Alternative Herbicides for Control of Glyphosate-Resistant Giant Ragweed in Nebraska
}

\author{
Stevan Z. Knezevic ${ }^{1}$, O. Adewale Osipitan ${ }^{1}$, Jon E. Scott ${ }^{1}, \&$ Dejan Nedeljkovic ${ }^{2}$ \\ ${ }^{1}$ Northeast Research and Extension Center, Haskell Agricultural Laboratory, University of Nebraska-Lincoln, \\ Concord, NE, USA \\ ${ }^{2}$ Faculty of Agriculture, University of Belgrade, Belgrade, Serbia \\ Correspondence: Stevan Z. Knezevic, Haskell Agricultural Laboratory, University of Nebraska-Lincoln, \\ Concord, NE, USA. Tel: 1-402-584-3810. E-mail: sknezevic2@unl.edu
}

Received: September 21, 2018 Accepted: October 11, 2018 Online Published: November 7, 2018

doi:10.5539/sar.v8n1p21 URL: https://doi.org/10.5539/sar.v8n1p21

\begin{abstract}
Giant ragweed is an early emerging and one of the most competitive summer annual species found in many fields throughout North America. Extensive use of glyphosate in glyphosate-tolerant (GT) crops has evolved giant ragweed populations with glyphosate resistance. Field dose-response studies were conducted to determine the influence of growth stage on the level of glyphosate resistance in a suspected giant ragweed population. In addition, efficacy of alternative pre-plant, pre-emergence (PRE) and post-emergence (POST) herbicides were evaluated in corn and soybeans for glyphosate-resistant (GR) giant ragweed control. The field glyphosate dose-response studies confirmed that the suspected giant ragweed population were resistant ranging from 14- to 32 -fold resistance depending on the growth stage of glyphosate application. The 10,20 and $30 \mathrm{~cm}$ tall giant ragweed had 14, 17 and 32X resistance level, respectively. The dose-response studies indicated that the 10, 20, and $30 \mathrm{~cm}$ tall GR giant ragweed was controlled $90 \%$ with 214, 402 and $482 \mathrm{~g}$ ae ha ${ }^{-1}$ of dicamba, respectively, when tank-mixed with glyphosate $\left(1060 \mathrm{~g}_{\text {ae }} \mathrm{ha}^{-1}\right) 21$ days after treatment (DAT). All evaluated pre-plant herbicides for corn provided $\geq 90 \%$ control of the GR giant ragweed at 30 DAT; among which the best control $(100 \%)$ was achieved with pre-plant application of atrazine $\left(2240 \mathrm{~g}^{3} \mathrm{ha}^{-1}\right)$, isoxaflutole $\left(90 \mathrm{~g}\right.$ ai ha $\left.{ }^{-1}\right)$, and premix of flumioxazin/pyroxasulfone ( $315 \mathrm{~g}$ ai ha $\left.^{-1}\right)$. Herbicide combinations of different site of action provided greater than $90 \%$ control of the GR giant ragweed population in a PRE followed by POST herbicide program in corn and soybean, suggesting that alternative herbicide for giant ragweed control are available.
\end{abstract}

Keywords: corn, soybean, glyphosate resistance, giant ragweed, herbicides

\section{Introduction}

Giant ragweed (Ambrosia trifida L.) is a broadleaf weed species found in many fields in North America (Regnier et al., 2016). Giant ragweed is a summer annual weeds in field crops such as soybean and corn in Nebraska, with emergence beginning in March (Kaur et al., 2016). The early emergence, fast growth as well as the ability to survive in adverse environment have been attributed to the high competitive advantage of giant ragweed against field crops (Ganie et al., 2016; Goplen et al., 2017). Studies have shown that giant ragweed is very competitive in field crops even with low densities (Ganie et al., 2016; Harrison et al., 2001).

The control of giant ragweed in field crops over the last three decades has been more difficult due to development of herbicide resistant biotypes. For example, extensive use of acetolactate synthase (ALS) inhibitors led to the development of ALS-resistant giant ragweed in the 1990s (Heap, 2018; Schrage, 2018). In the more recent history, widespread adoption of glyphosate-tolerant (GT) crops and repeated use of glyphosate for both burndown and post-emergence weed control (Regnier et al., 2016) resulted in many glyphosate resistant species, including giant ragweed (Kaur et al., 2014; Heap \& Duke, 2018).

Giant ragweed control using herbicides with multiple modes of action would reduce risk of weed resistance. Alternative herbicides such as auxin herbicides, photosystem II inhibitors, hydroxyphenylpyruvate dioxygenase (HPPD) inhibitors, and protoporphyrinogen oxidase (PPO) inhibitors had shown to be effective for giant ragweed control (Riley \& Bradley, 2014; Belfry \& sikkema, 2015; Mahoney et al., 2015; Wuerffel et al., 2015; Ditschun et al., 2016; Goplen et al., 2018; Osipitan et al., 2018). Therefore, field studies were conducted in 
Nebraska to determine the level of glyphosate resistance in a suspected glyphosate-resistant (GR) giant ragweed population and to evaluate alternative herbicides for pre-plant, pre-emergence (PRE) and post-emergence (POST) herbicides in corn and soybeans for GR giant ragweed control.

\section{Materials and Methods}

\subsection{Field Dose-Response Studies}

Field experiments were conducted in 2012 at two locations with a natural infestation of suspected GR giant ragweed located near David City, NE (41.258N, 97.138W). The study was laid out in a randomized complete block design with three replications for dose response studies on glyphosate alone, dicamba tank-mixed with glyphosate, or saflufenacil tank-mixed with glyphosate at three growth stages $(10,20$, and $30 \mathrm{~cm}$ tall plants) of giant ragweed. The dose response studies consisted of four doses of glyphosate alone $(1060,4240,8500$, and $\left.17000 \mathrm{~g} \mathrm{ae} \mathrm{ha}^{-1}\right)$; four doses of saflufenacil $\left(12.5,25,50\right.$ and $100 \mathrm{~g}$ ai ha $\left.{ }^{-1}\right)$ tank-mixed with a recommended dose of glyphosate $\left(1060 \mathrm{~g}\right.$ ae ha $\left.\mathrm{h}^{-1}\right)$; and four doses of dicamba $\left(210,420,840\right.$ and $1680 \mathrm{~g}^{2}$ ai ha $\left.{ }^{-1}\right)$ mixed with a recommended dose of glyphosate $\left(1060 \mathrm{~g}_{\text {ae }} \mathrm{ha}^{-1}\right)$; as well as a non-treated control. Each experimental plot was 8 $\mathrm{m}$ long by $3 \mathrm{~m}$ wide and was planted with two rows of corn and two rows of soybean on May 21, 2012 in $76 \mathrm{~cm}$ rows with a four-row planter. The first application of herbicide was made when giant ragweed plants were $10 \mathrm{~cm}$ tall as early POST (EPOST), the second application to $20 \mathrm{~cm}$ tall plants, at mid-POST (MPOST), and the third application to $30 \mathrm{~cm}$ tall plants as late POST (LPOST).

Herbicide treatments were applied with a $\mathrm{CO}_{2}$-pressurized backpack sprayer calibrated to deliver $140 \mathrm{~L} \mathrm{ha}^{-1}$ solution at $172 \mathrm{kPa}$ with TeeJet XR 110015 flat-fan nozzles at a speed of $4.3 \mathrm{~km} \mathrm{~h}^{-1}$. The GR giant ragweed control was visually rated at 7, 14 and 21 days after treatment (DAT) on a scale of $0 \%$ (no control) to $100 \%$ (complete control). At 21 DAT, the giant ragweed plants within $0.25 \mathrm{~m}^{2}$ quadrat randomly placed in each plot were severed at the soil surface and oven-dried at $35 \mathrm{C}$ for 7 days, then shoot biomass was measured. The shoot biomass reduction (\%) was expressed as:

$$
\text { Shoot biomass reduction }(\%)=[(G-H) / G] \times 100
$$

Where $G$ was the biomass (above ground) of giant ragweed plants in non-treated plots and $H$ was the biomass of giant ragweed plants in the respective herbicide treated plots.

A four-parameter log-logistic model was best to relate giant ragweed responses (visual control and biomass reduction) to herbicide treatments using "drc" package in R statistical software (R Development Core Team, 2018; Knezevic et al., 2007). The model was given as:

$$
Y=C+\{D-C / 1+\exp [B(\log X-\log E)]\}
$$

where $\mathrm{Y}$ was the giant ragweed response, $C$ was the lower limit, $D$ was the upper limit, $X$ was the herbicide dose, $E$ was the dose resulting to $50 \%$ response between the upper and lower limit (also known as $\mathrm{ED}_{50}$ ), $B$ was the slope around $E$. The effective dose needed to suppress the giant ragweed population by $50 \%\left(\mathrm{ED}_{50}\right)$ and $90 \%$ $\left(\mathrm{ED}_{90}\right)$ was estimated from the above model (equation 2). Differences between the ED values for each growth stage and herbicide treatment were determined by the standard errors (SE) (Knezevic et al., 2018).

\subsection{Evaluation of Alternative Herbicide Programs on GR Giant Ragweed}

Field experiments were conducted in 2013 and 2014, near David City, NE to evaluate control of GR giant ragweed with pre-plant, and PRE followed by (fb) POST herbicides in corn; as well as PRE fb POST and POST-only in soybean. The soil texture of the location was silty clay loam (20\% sand, $54 \%$ silt, $38 \%$ clay) with a pH of 5.7, and 2.2\% organic matter. Total rainfall from April to October was $67.3 \mathrm{~cm}$ in 2013 and $61.0 \mathrm{~cm}$ in 2014. Average daily temperature was 23 and $25^{\circ} \mathrm{C}$ in 2013 and 2014, respectively. Glyphosate-tolerant corn (H-9138, Golden Harvest Seeds, Waterloo, NE 68069) was planted at 69,780 seeds ha ${ }^{-1}$ in rows spaced $76 \mathrm{~cm}$ apart on June 12, 2013 and June 5, 2014. Glyphosate-tolerant soybean (92Y70, Pioneer Seed, Allen, NE 68710) was planted at 360,760 seeds ha ${ }^{-1}$ also in rows spaced $76 \mathrm{~cm}$ apart on May 18, 2013 and May 28, 2014. Each of the herbicide programs has 12 treatments except POST-only (5 treatments) in soybean (Table 1, 2, 3 and 4). Experiments were conducted in a randomized complete block design with three replications for each of the program in corn and soybean. Individual plots were $8 \mathrm{~m}$ long and $3 \mathrm{~m}$ wide.

Herbicide applications were made using a $\mathrm{CO}_{2}$-pressurized backpack boom sprayer calibrated to deliver $140 \mathrm{~L}$ $\mathrm{ha}^{-1}$ at $172 \mathrm{kPa}$ (for PRE) and $276 \mathrm{kPa}$ (for Pre-plant and POST), through four AIXR 11002 (for PRE) and 10015 (for Pre-plant and POST) nozzle tips (Turbo TeeJet, Spraying systems Co., P.O. Box 7900, Wheaton, IL 60187) with a boom length of $200 \mathrm{~cm}$. The Pre-plant herbicides applications were conducted in April prior to planting of corn or soybean in May or June. The PRE herbicides were applied immediately after planting corn or soybean, 
while POST herbicides were applied when the GR giant ragweed plants were 8 to $11 \mathrm{~cm}$ tall.

Visually rated weed control on the scale of 0 (no control) to $100 \%$ (complete control) were collected at 30 , 60 and 75 DAT for pre-plant; 30 days after PRE (DAPRE) and 30 days after POST (DAPOST) for PRE fb POST; 30 DAT for POST-only. GR giant ragweed biomass was collected from plants within $0.25 \mathrm{~m}^{2}$ quadrats placed the middle two corn or soybean rows in each plot at 30 DAT for all herbicide programs. Biomass reduction (\%) was calculated as shown in equation 1 .

An initial test of normality of data using the PROC Univariate procedure in SAS v. 9.4 software (SAS Institute, Cary, NC 27513) suggested that the collected data did not follow a normal distribution. Hence, data were arcsine transformed to reduce the heterogeneity of treatment variances. Tests of significance of treatments on GR giant ragweed population was conducted with ANOVA using the PROC MIXED procedure in SAS, with replicates and years considered random variables. The untreated plot data were excluded from the analyses of the visually rated GR giant ragweed control. If ANOVA indicated significant treatment effects, means of the transformed data were separated with Fisher's protected LSD test at $\mathrm{P} \leq 0.05$; however, back-transformed data were presented in tables for easy interpretation.

Table 1. List of pre-plant herbicides used for control of GR Giant Ragweed in Corn in 2013 and 2014

\begin{tabular}{|c|c|c|c|c|}
\hline Herbicides & Trade name & Rate & Manufacturer & Adjuvant \\
\hline Atrazine & Aatrex ${ }^{\circledast}$ & $\begin{array}{l}\text { g ai ha }{ }^{-1} \\
2240\end{array}$ & $\begin{array}{l}\text { Syngenta Crop Protection, Inc., } \\
\text { Greensboro, NC } 27419\end{array}$ & $\mathrm{COC}$ \\
\hline Isoxaflutole & Balance Flexx ${ }^{\circledast}$ & 90 & $\begin{array}{l}\text { Bayer CropScience, Research } \\
\text { Triangle Park, NC } 27709\end{array}$ & MSO \\
\hline Isoxaflutole + Atrazine & $\begin{array}{l}\text { Balance Flexx }{ }^{\circledR}+ \\
\text { Aatrex }^{\circledR}\end{array}$ & $90+1120$ & $\begin{array}{l}\text { Bayer CropScience }+ \text { Syngenta } \\
\text { Crop Protection }\end{array}$ & $\mathrm{MSO}+\mathrm{AMS}$ \\
\hline Mesotrione & Callisto ${ }^{\circledR}$ & 300 & Syngenta Crop Protection & AMS \\
\hline Thiencarbazone-methyl/isoxaflutole & Corvus $^{\circledR}$ & 129 & Bayer CropScience & $\mathrm{AMS}+\mathrm{COC}$ \\
\hline Flumioxazin/Pyroxasulfone & Fierce $^{\circledR}$ & 315 & $\begin{array}{l}\text { Valent USA Corporation, Walnut } \\
\text { Creek, CA } 94596\end{array}$ & AMS \\
\hline Dimethenamid-P/atrazine & Guardsman Max ${ }^{\circledR}$ & 723 & BASF Corporation & $\mathrm{COC}$ \\
\hline S-metolachlor/mesotrione/atrazine & Lumax EZ $\mathrm{E}^{\circledast}$ & 2780 & Syngenta Crop Protection & AMS \\
\hline Saflufenacil & Sharpen ${ }^{\circledR}$ & 75 & BASF Corporation & AMS \\
\hline Flumioxazin + Atrazine & $\begin{array}{l}\text { Valor }^{\circledR}+ \\
\text { Aatrex }^{\circledR}\end{array}$ & $210+1120$ & Valent USA Corporation & $\mathrm{AMS}+\mathrm{COC}$ \\
\hline Saflufenacil/dimethenamid-P & Verdict $^{\circledR}$ & 730 & BASF Corporation & AMS \\
\hline Mesotrione + S-metolachlor & Zemax $^{\circledR}$ & 600 & Syngenta Crop Protection & AMS \\
\hline
\end{tabular}

${ }^{a} A$ bbreviations: Herbicide premix (/); herbicide tank-mix (+ )

${ }^{\mathrm{b}}$ AMS, ammonium sulfate (DSM Chemicals North America Inc., Augusta, GA); COC, crop oil concentrate (Agridex®, Helena Chemical Co., Collierville, TN); MSO, methylated seed oil (Noble ${ }^{\circledR}$, Winfield Solutions, Shoreview, MN). 
Table 2. List of PRE followed by (fb) POST herbicides used for control of GR giant ragweed in corn in 2013 and 2014

\begin{tabular}{|c|c|c|c|c|}
\hline Herbicide & Trade name & Rate & Manufacturer & Adjuvant \\
\hline & & $\mathrm{g}$ ai $(\mathrm{ae}) \mathrm{ha}^{-1}$ & & \\
\hline Atrazine fb 2,4-D & Aatrex ${ }^{\circledR}$ fb 2,4-D & $\begin{array}{ll}2240 & \mathrm{fb} \\
535 & \end{array}$ & $\begin{array}{l}\text { Sygenta Crop Protection, Inc., Greensboro, } \\
\text { NC } 27419 \text { fb Winfield Solutions, LLC, } \\
\text { St. Paul, MN } 55164\end{array}$ & $\mathrm{COC}+\mathrm{NIS}$ \\
\hline Isoxaflutole fb 2,4-D & $\begin{array}{l}\text { Balance Flexx }{ }^{\circledR} \text { fb } \\
\text { 2,4-D }\end{array}$ & $90 \mathrm{fb} 535$ & $\begin{array}{l}\text { Bayer CropScience, Research Triangle Park, } \\
\text { NC } 27709 \text { fb Winfield Solutions }\end{array}$ & MSO + NIS \\
\hline Isoxaflutole + atrazine fb 2,4-D & $\begin{array}{l}\text { Balance Flexx }{ }^{\circledR}+ \\
\text { Atrazine }^{\circledR} \text { fb 2,4-D }\end{array}$ & $\begin{array}{l}90+1120 \\
\mathrm{fb} 535\end{array}$ & $\begin{array}{l}\text { Bayer CropScience }+ \text { Sygenta Crop } \\
\text { Protection fb Winfield Solutions }\end{array}$ & $\begin{array}{l}\mathrm{MSO}+\mathrm{COC} \\
+\mathrm{NIS}\end{array}$ \\
\hline $\begin{array}{l}\text { Mesotrione fb flumetsulam/ } \\
\text { clopyralid }\end{array}$ & $\begin{array}{l}\text { Callisto }^{\circledR} \\
\text { Hornet }^{\circledR}\end{array}$ & $300 \mathrm{fb} 54$ & $\begin{array}{l}\text { Syngenta Crop Protection fb Dow } \\
\text { AgroSciences LLC, } 9330 \text { Zionsville Road, } \\
\text { Indianapolis, IN } 46268\end{array}$ & AMS \\
\hline $\begin{array}{l}\text { Thiencarbazone-methyl/isoxaflutole } \\
\mathrm{fb} 2,4-\mathrm{D}\end{array}$ & Corvus $^{\circledast}$ fb 2,4-D & $129 \mathrm{fb} 535$ & $\begin{array}{l}\text { Bayer CropScience fb } \\
\text { Winfield Solutions }\end{array}$ & $\mathrm{MSO}+\mathrm{NIS}$ \\
\hline 2,4 D (POST-only) & 2,4-D & 535 & Winfield Solutions & NIS \\
\hline Dimethenamid-P/atrazine fb 2,4-D & $\begin{array}{l}\text { Guardsman } \operatorname{Max}^{\circledR} \\
\text { fb 2,4-D }\end{array}$ & $723 \mathrm{fb} 535$ & $\begin{array}{l}\text { BASF Corporation fb } \\
\text { Winfield Solutions }\end{array}$ & $\mathrm{COC}+\mathrm{NIS}$ \\
\hline $\begin{array}{l}\text { S-metolachlor/mesotrione/atrazine } \\
\mathrm{fb} 2,4-\mathrm{D}\end{array}$ & $\begin{array}{l}\operatorname{Lumax} \quad \mathrm{EZ}^{\circledR} \quad \mathrm{fb} \\
2,4-\mathrm{D}\end{array}$ & $\begin{array}{l}2780 \quad \mathrm{fb} \\
535\end{array}$ & Sygenta Crop Protection & \\
\hline $\begin{array}{l}\text { Saflufenacil fb diflufenzopyr/ } \\
\text { dicamba }\end{array}$ & $\begin{array}{l}\text { Sharpen }{ }^{\circledR} \\
\text { Distinct }^{\circledR}\end{array}$ & $75 \mathrm{fb} 360$ & BASF Corporation & \\
\hline $\begin{array}{l}\text { Saflufenacil/dimethenamid-P fb } \\
\text { diflufenzopyr/dicamba }\end{array}$ & $\begin{array}{l}\text { Verdict }^{\circledR} \\
\text { Status }^{\circledR}\end{array}$ & $730 \mathrm{fb} 360$ & BASF Corporation & MSO + AMS \\
\hline $\begin{array}{l}\text { Mesotrione }+ \text { S-metolachlor } \mathrm{fb} \\
\text { diflufenzopyr/dicamba }\end{array}$ & Zemax $^{\circledast}$ fb Status ${ }^{\circledast}$ & $600 \mathrm{fb} 360$ & $\begin{array}{l}\text { Sygenta Crop Protection } \mathrm{fb} \\
\text { BASF Corporation }\end{array}$ & $\mathrm{MSO}+\mathrm{AMS}$ \\
\hline
\end{tabular}

${ }^{\text {a} A b b r e v i a t i o n s: ~ H e r b i c i d e ~ p r e m i x ~(/) ; ~ h e r b i c i d e ~ t a n k-m i x ~(+~) ; ~ f b, ~ f o l l o w e d ~ b y . ~}$

${ }^{\mathrm{b}}$ AMS, ammonium sulfate (DSM Chemicals North America Inc., Augusta, GA); COC, crop oil concentrate (Agridex®, Helena Chemical Co., Collierville, TN); NIS, nonionic surfactant (Induce ${ }^{\circledR}$, Helena Chemical Co., Collierville, TN), MSO, methylated seed oil (Noble ${ }^{\circledR}$, Winfield Solutions, Shoreview, MN). 
Table 3. List of PRE followed by (fb) POST herbicides used for control of GR giant ragweed in soybean in 2013 and 2014

\begin{tabular}{|c|c|c|c|c|c|}
\hline Herbicide & Trade name & Rate & Manufacturer & \multicolumn{2}{|c|}{ Adjuvant } \\
\hline $\begin{array}{l}\text { Sulfentrazone/cloransulam fb } \\
\text { imazamox + acifluorfen }\end{array}$ & $\begin{array}{l}\text { Authority First }{ }^{\circledR} \text { fb } \\
\text { Raptor }^{\circledR}+\text { Ultra } \\
\text { Blazer }^{\circledR}\end{array}$ & $\begin{array}{l}\text { g ai (ae) ha- } \\
392 \mathrm{fb} 35 \\
+280\end{array}$ & $\begin{array}{l}\text { FMC Corporations, Philadelphia, PA } 19103 \text { fb BASF } \\
\text { Corporation + United Phosphorus, Inc, } \\
\text { King of Prussia, PA } 19406\end{array}$ & $\begin{array}{l}\text { AMS } \\
\text { COC }\end{array}$ & + \\
\hline $\begin{array}{l}\text { Chlorimuron/flumioxazin/ } \\
\text { thifensulfuron } \\
\text { fb imazamox }+ \text { acifluorfen }\end{array}$ & $\begin{array}{l}\text { Envive }^{\circledR} \text { fb Raptor }^{\circledR} \\
+ \text { Ultra Blazer }^{\circledR}\end{array}$ & $\begin{array}{l}106 \mathrm{fb} 35 \\
+280\end{array}$ & $\begin{array}{l}\text { DuPont, Wilmington, DE } 19898 \text { fb BASF Corporation } \\
\text { + United Phosphorus, Inc, } \\
\text { King of Prussia, PA } 19406\end{array}$ & $\mathrm{COC}$ & \\
\hline Clorasulam fb lactofen & $\begin{array}{l}\text { FirstRate }^{\circledR} \\
\text { Phoenix }^{\circledR}\end{array}$ & $10 \mathrm{fb} 219$ & $\begin{array}{l}\text { Dow AgroSciences LLC, } 9330 \text { Zionsville Road, } \\
\text { Indianapolis, IN } 46268 \text { fb Valent USA Corporation }\end{array}$ & AMS & \\
\hline $\begin{array}{l}\text { Flumioxazin/clorasulam }+ \\
\text { flumioxazin }+ \\
\text { clorasulam fb imazamox }+ \\
\text { acifluorfen }\end{array}$ & $\begin{array}{l}\text { Gangster }^{\circledR}+ \\
\text { Valor SX }^{\circledR}+ \\
\text { FirstRate }^{\circledR} \text { fb } \\
\text { Raptor }^{\circledR}+\text { Ultra } \\
\text { Blazer }^{\circledR}\end{array}$ & $\begin{array}{l}129+75+ \\
10 \mathrm{fb} 35+ \\
280\end{array}$ & $\begin{array}{l}\text { Valent USA Corporation, Walnut Creek, CA } 94596+ \\
\text { Valent + Dow AgroSciences fb BASF Corporation + } \\
\text { United Phosphorus, Inc, King of Prussia, PA } 19406\end{array}$ & $\begin{array}{l}\text { COC } \\
\text { AMS }\end{array}$ & + \\
\hline $\begin{array}{l}\text { Saflufenacil/imazethapyr } \mathrm{fb} \\
\text { lactofen }\end{array}$ & $\begin{array}{l}\text { Op } \quad \text { Till }^{\circledR} \\
\text { Phoenix }^{\circledR}\end{array}$ & $100 \mathrm{fb} 219$ & $\begin{array}{l}\text { BASF Canada Inc. } 100 \text { Milverton } \\
\text { Drive, 5th Floor Mississauga, } \\
\text { ON fb Valent Corporation }\end{array}$ & AMS & \\
\hline Imazethapyr fb lactofen & $\begin{array}{l}\text { Pursuit }^{\circledR} \\
\text { Phoenix }^{\circledR}\end{array}$ & 70 fb 219 & $\begin{array}{l}\text { BASF Corporation } \mathrm{fb} \\
\text { Valent Corporation }\end{array}$ & $\begin{array}{l}\text { MSO } \\
\text { AMS }\end{array}$ & + \\
\hline $\begin{array}{l}\text { Imazethapyr }+ \text { S-metolachlor } \\
\text { fb lactofen }\end{array}$ & $\begin{array}{l}\text { Pursuit }^{\circledR}+ \\
\text { Dual II Magnum }{ }^{\circledR} \\
\text { fb Phoenix }{ }^{\circledR}\end{array}$ & $\begin{array}{l}70+1411 \\
\mathrm{fb} 219\end{array}$ & $\begin{array}{l}\text { BASF Corporation }+ \text { Sygenta Crop } \\
\text { Protection fb Valent Corporation }\end{array}$ & $\begin{array}{l}\text { MSO } \\
\text { AMS }\end{array}$ & + \\
\hline Imazaquin fb lactofen & $\begin{array}{l}\text { Scepter }^{\circledR}+\text { Dual II } \\
\text { Magnum }^{\circledR} \quad \mathrm{fb} \\
\text { Phoenix }^{\circledR}\end{array}$ & $\begin{array}{lr}143 & + \\
1411 & f b \\
219 & \end{array}$ & $\begin{array}{l}\text { AMVAC Chemical Corporation, Los Angeles, CA } \\
90023+\text { Sygenta Crop Protection fb Valent Corporation }\end{array}$ & $\begin{array}{l}\text { NIS } \\
\text { AMS }\end{array}$ & + \\
\hline Metribuzin fb lactofen & $\begin{array}{l}\text { Sencor }^{\circledR} \\
\text { Phoenix }^{\circledR}\end{array}$ & 438 fb 219 & $\begin{array}{l}\text { Bayer CropScience, Research Triangle } \\
\text { Park, NC } 27709 \mathrm{fb} \text { Valent Corporation }\end{array}$ & - & \\
\hline Flumioxazin fb clorasulam & $\begin{array}{l}\text { Valor } \mathrm{SX}^{\circledR} \mathrm{fb} \\
\text { FirstRate }^{\circledR}\end{array}$ & $113 \mathrm{fb} 5$ & Valent USA Corporation fb Dow AgroSciences & $\mathrm{COC}$ & \\
\hline $\begin{array}{l}\text { Flumioxazin/Chlorimuron fb } \\
\text { imazamox + acifluorfen }\end{array}$ & $\begin{array}{l}\text { Valor } \text { XLT }^{\circledR} \text { fb } \\
\text { Raptor }^{\circledR}+\text { Ultra } \\
\text { Blazer }^{\circledR}\end{array}$ & $\begin{array}{l}158 \mathrm{fb} 35 \\
+280\end{array}$ & $\begin{array}{l}\text { Valent USA Corporation fb BASF } \\
\text { Corporation + United Phosphorus }\end{array}$ & $\begin{array}{l}\text { COC } \\
\text { AMS }\end{array}$ & + \\
\hline
\end{tabular}

${ }^{a}$ Abbreviations: Herbicide premix (/); herbicide tank-mix (+ ); fb, followed by.

${ }^{\mathrm{b}}$ AMS, ammonium sulfate (DSM Chemicals North America Inc., Augusta, GA); COC, crop oil concentrate (Agridex®, Helena Chemical Co., Collierville, TN); NIS, nonionic surfactant (Induce ${ }^{\circledR}$, Helena Chemical Co., Collierville, TN), MSO, methylated seed oil (Noble ${ }^{\circledR}$, Winfield Solutions, Shoreview, MN).

Table 4. List of POST-only herbicides used for control of GR giant ragweed in soybean in 2013 and 2014

\begin{tabular}{|c|c|c|c|c|}
\hline Herbicide & Trade name & Rate & Manufacturer & Adjuvant \\
\hline Clorasulam & FirstRate ${ }^{\circledR}$ & $\begin{array}{l}\text { g ai ha }{ }^{-1} \\
5\end{array}$ & $\begin{array}{l}\text { Dow AgroSciences LLC, } 9330 \\
\text { Zionsville Road, Indianapolis, IN } 46268\end{array}$ & $\mathrm{COC}$ \\
\hline $\begin{array}{l}\text { Thifensulfuron + } \\
\text { chlorimuron }\end{array}$ & $\begin{array}{l}\text { Harmony SG }{ }^{\circledR}+ \\
\text { Classic }^{\circledR}\end{array}$ & $5+5$ & $\begin{array}{l}\text { DuPont } 4417 \text { Lancaster Pike Wilmington, } \\
\text { DE } 19805 \text {, USA }\end{array}$ & $\mathrm{COC}+\mathrm{AMS}$ \\
\hline Lactofen & Phoenix ${ }^{\circledR}$ & 219 & Valent USA Corporation, Walnut Creek, CA 94596 & $\mathrm{COC}$ \\
\hline Imazamox + acifluorfen & Raptor $^{\circledR}+$ Ultra Blazer $^{\circledR}$ & $35+280$ & $\begin{array}{l}\text { BASF Corporation + United Phosphorus, } \\
\text { Inc, King of Prussia, PA } 19406\end{array}$ & $\mathrm{COC}+\mathrm{AMS}$ \\
\hline Fomesafen/glyphosate & Flexstar GT $^{\circledR}$ & 1382 & Sygenta Crop Protection & AMS \\
\hline
\end{tabular}

${ }^{a}$ Abbreviations: Herbicide premix (/); herbicide tank-mix (+ )

${ }^{\mathrm{b}}$ AMS, ammonium sulfate (DSM Chemicals North America Inc., Augusta, GA); COC, crop oil concentrate (Agridex®, Helena Chemical Co., Collierville, TN) 

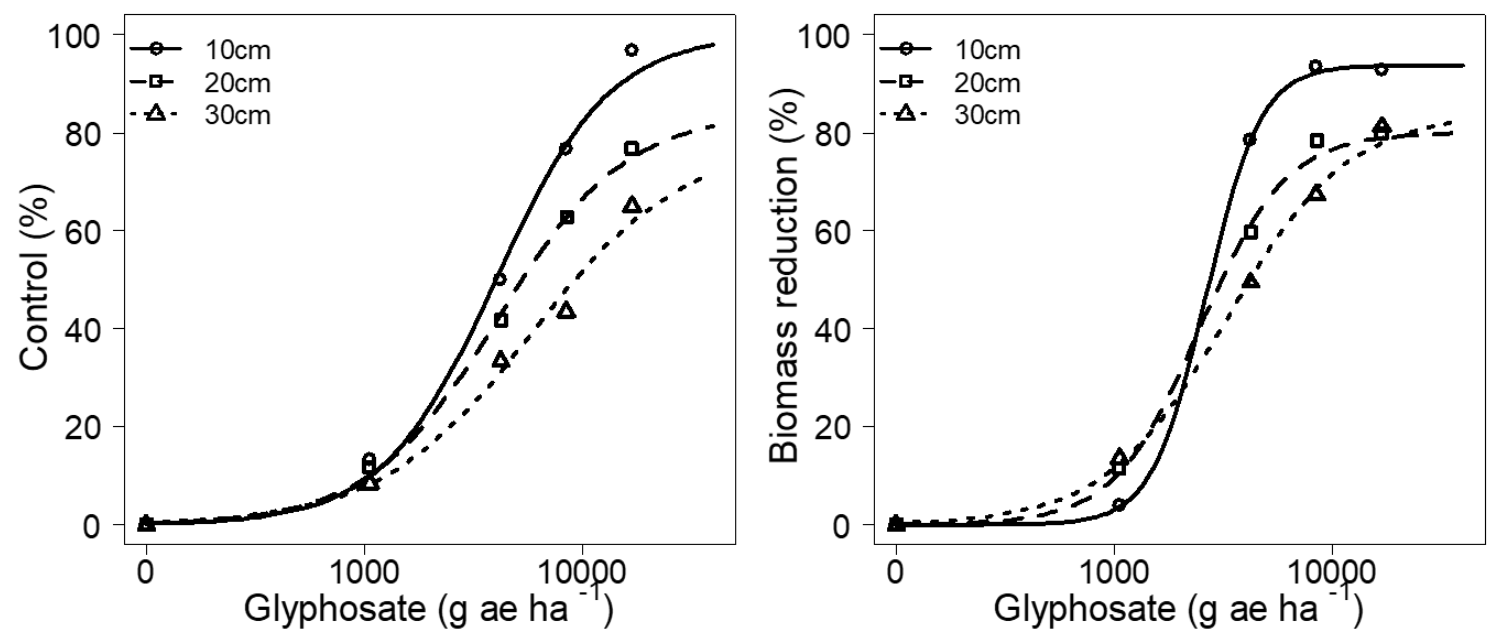

Figure 1. Control and biomass reduction of 10, 20 or $30 \mathrm{~cm}$ tall giant ragweed at 21 days after treatment (DAT) with POST-applied glyphosate in field dose-response studies

\section{Results and Discussion}

\subsection{Field Dose-Response Studies}

The field dose-response studies indicates that the giant ragweed population was resistant to glyphosate and that the level of resistance was influenced by the growth stage of glyphosate application (Figure 1 and Table 5). The resistance level was calculated by dividing the effective doses (ED values) of glyphosate for 50 or $90 \%$ control (or biomass reduction) by the label rate $\left(1060 \mathrm{~g}_{\text {ae }} \mathrm{ha}^{-1}\right)$. Based on control rating, the $\mathrm{ED}_{90}$ values at $21 \mathrm{DAT}$ for 10, 20, and $30 \mathrm{~cm}$ tall giant ragweed were 15212,18071, and $34042 \mathrm{~g}$ ae ha $^{-1}$, respectively. The corresponding levels of glyphosate resistance (based on the $\mathrm{ED}_{90}$ ) for 10, 20, and $30 \mathrm{~cm}$ tall giant ragweed at 21 DAT was 14X, 17X, and $32 \mathrm{X}$, respectively. Similar glyphosate resistance levels were obtained in dose-response curves based on biomass reduction (Figure 1; Table 5). Based on biomass reduction, the GR giant ragweed was most resistant to glyphosate when plant was $30 \mathrm{~cm}$ tall (32X), followed by $20 \mathrm{~cm}$ tall $(16 \mathrm{X})$ and $10 \mathrm{~cm}$ tall $(13 \mathrm{X})$. These results suggest that resistance level of the GR giant ragweed population increased with increase in plant size. Varying levels of a weed species resistance to glyphosate at different growth stages have been previously reported (Koger et al., 2004; Shrestha et al., 2007; VanGessel, 2001; Norsworthy et al., 2010).
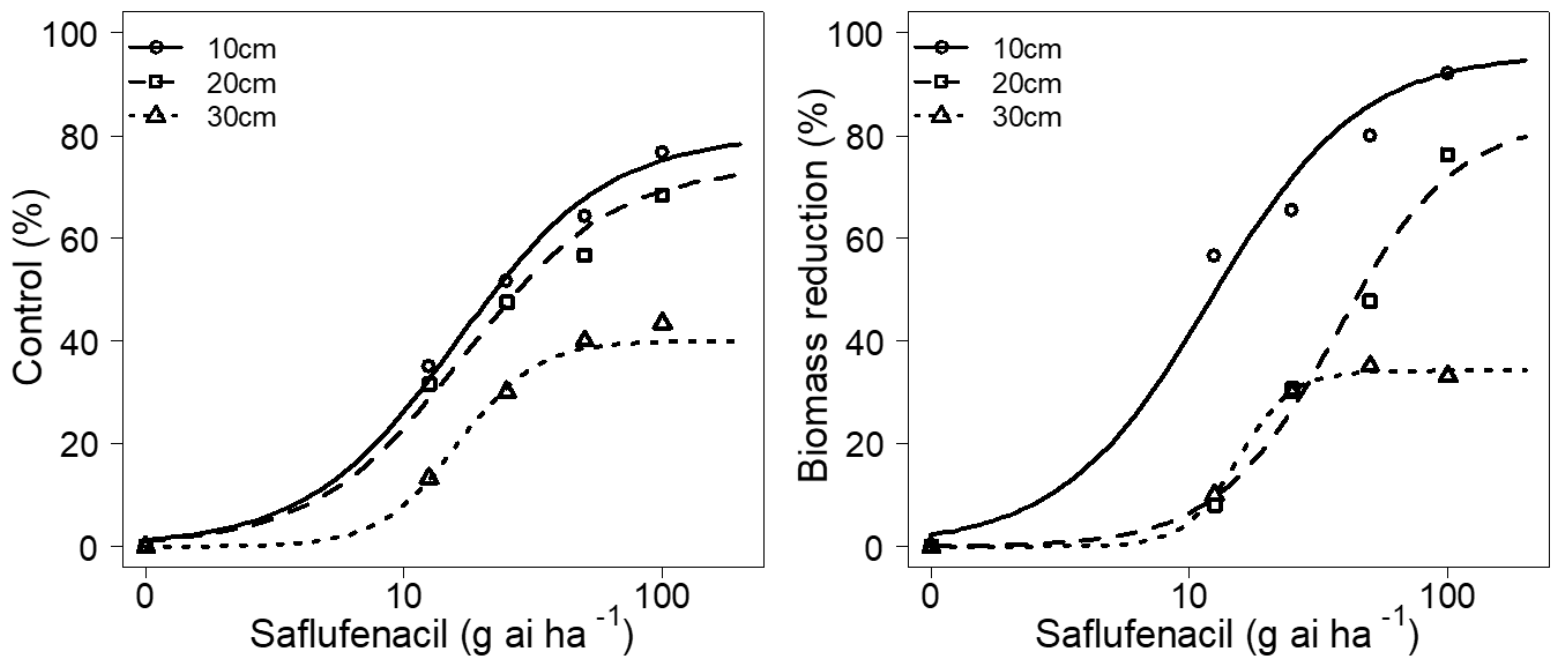

Figure 2. Control and biomass reduction of 10,20 or $30 \mathrm{~cm}$ tall giant ragweed at 21 days after treatment (DAT) with POST-applied saflufenacil tank-mixed with glyphosate $\left(1060 \mathrm{~g}\right.$ ae ha $\left.{ }^{-1}\right)$ in field dose-response studies 

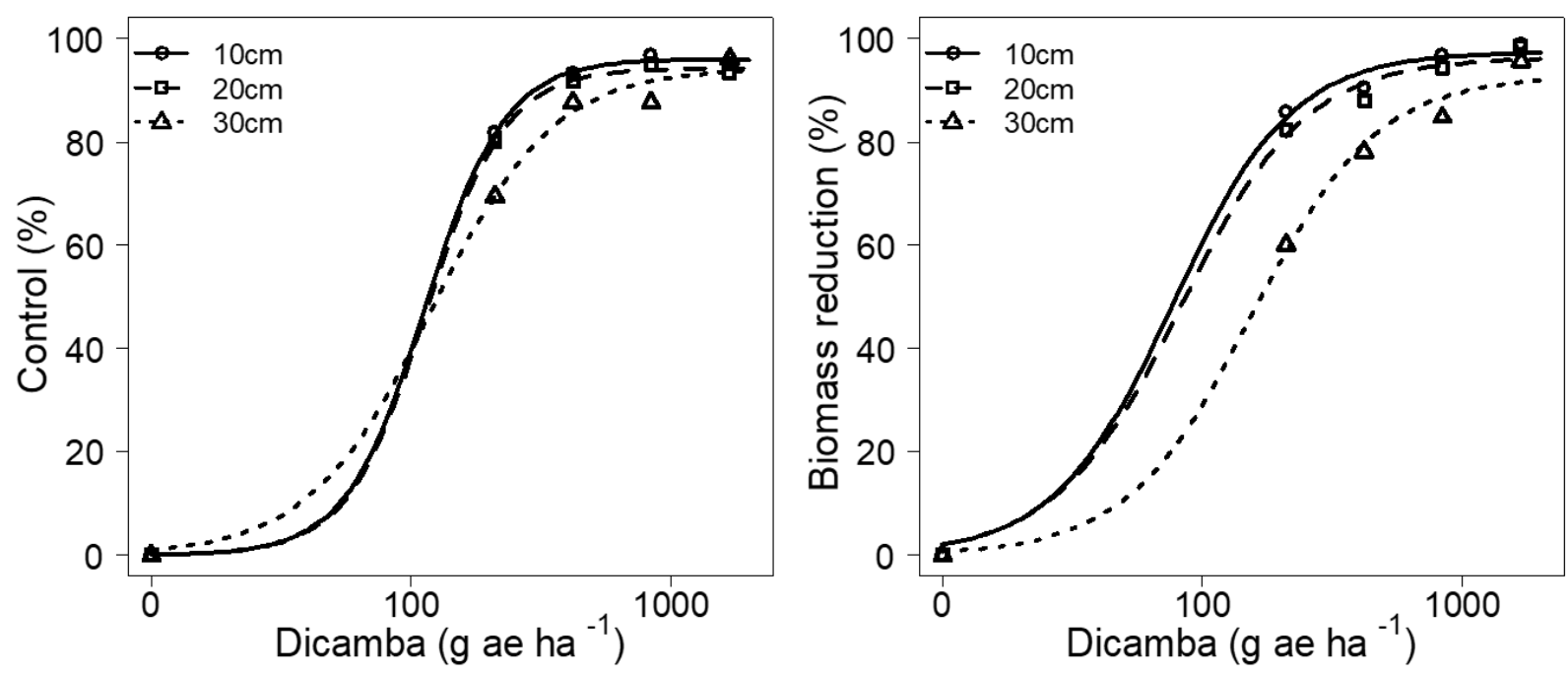

Figure 3. Control and biomass reduction of 10,20 or $30 \mathrm{~cm}$ tall giant ragweed at 21 days after treatment (DAT) with POST-applied dicamba tank-mixed with glyphosate $\left(1060 \mathrm{~g} \mathrm{ae} \mathrm{ha}^{-1}\right)$ in field dose-response studies

The dose-response of the GR giant ragweed to saflufenacil tank-mixed with glyphosate (1060 $\mathrm{g}$ ae ha-) showed a poor control (Figure 2; Table 6). For example, a label rate of saflufenacil (25 $\mathrm{g}$ ai ha $\left.{ }^{-1}\right)$ tank-mixed with glyphosate $\left(1060 \mathrm{~g}\right.$ ae ha $\left.^{-1}\right)$ provided 48, 45 and $27 \%$ control of GR giant ragweed when plant was 10, 20 and 30 $\mathrm{cm}$ tall respectively, at 21 DAT. The $\mathrm{ED}_{90}$ indicated that $495-532 \mathrm{~g}$ ai ha $^{-1}$ of the saflufenacil tank-mixed with $1060 \mathrm{~g} \mathrm{ae} \mathrm{ha}^{-1}$ of glyphosate was needed to provide $90 \%$ control of the $10-20 \mathrm{~cm}$ tall GR giant ragweed (Table 6), which are much higher than the label rate $\left(25 \mathrm{~g} \mathrm{ai} \mathrm{ha}^{-1}\right)$. The $30 \mathrm{~cm}$ tall giant ragweed needed as much as 810 $\mathrm{g}$ ai ha ${ }^{-1}$ of saflufenacil to provide the same level of control. The estimated doses of saflufenacil for $90 \%$ biomass reduction were 238,437 , and $804 \mathrm{~g}_{\text {ai ha }}{ }^{-1}$ for 10,20 , and $30 \mathrm{~cm}$ tall giant ragweed, respectively. These results indicate that saflufenacil applied post-emergence is not a viable option for GR giant ragweed control.

Table 5. Effective doses of glyphosate at 21 DAT for $50 \%$ control or biomass reduction $\left(\mathrm{ED}_{50}\right)$ and $90 \%$ control or biomass reduction $\left(\mathrm{ED}_{90}\right)$ of 10,20 , or $30 \mathrm{~cm}$ tall giant ragweed

\begin{tabular}{|c|c|c|c|c|c|}
\hline Measure & Weed height $(\mathrm{cm})$ & $\mathrm{ED}_{50}( \pm \mathrm{SE})$ & $\mathrm{ED}_{90}( \pm \mathrm{SE})$ & \multicolumn{2}{|c|}{ Resistance level } \\
\hline \multirow[t]{4}{*}{ Control } & & -----Glyphos & $\mathrm{e}\left(\mathrm{g}\right.$ ae ha $\left.{ }^{-1}\right)$ & $\mathrm{ED}_{50}$ & $\mathrm{ED}_{90}$ \\
\hline & 10 & 4024 (103) & $15212(4246)$ & 4 & 14 \\
\hline & 20 & $4099(735)$ & 18071 (4012) & 4 & 17 \\
\hline & 30 & 5867 (1017) & $34042(12231)$ & 6 & 32 \\
\hline \multicolumn{6}{|c|}{ Biomass reduction } \\
\hline & 10 & $2757(357)$ & $14956(359)$ & 3 & 13 \\
\hline & 20 & $3119(602)$ & 17691 (1858) & 3 & 16 \\
\hline & 30 & 4215 (1210) & 34562 (4159) & 4 & 32 \\
\hline
\end{tabular}

However, the dose-response curve showed that tank-mix of dicamba and glyphosate provided a good control of the GR giant ragweed across all growth stages (Figure 3; Table 6). The required doses of dicamba for $90 \%$ control were 214, 402 and $482 \mathrm{~g}$ ae ha $^{-1}$ of dicamba for 10, 20, and $30 \mathrm{~cm}$ tall giant ragweed respectively, when tank-mixed with glyphosate $\left(1060 \mathrm{~g}^{2} \mathrm{~h} \mathrm{~h}^{-1}\right)$. The estimated doses for $90 \%$ control were within the recommended label rate $\left(560 \mathrm{~g} \mathrm{ae} \mathrm{ha}^{-1}\right)$ of dicamba, suggesting that GR giant ragweed can be effectively controlled by dicamba. Others also reported giant ragweed are known to be very susceptible to auxin herbicides including dicamba (Vink et al., 2012a; Robinson et al., 2012; Spaunhorst et al., 2014; Chahal et al., 2015). For example, Chahal et al. (2015) reported $93 \%$ control of 10 to $20 \mathrm{~cm}$ tall GR giant ragweed with 2,4-D, an auxnic herbicide; and giant ragweed was generally more susceptible to 2,4-D than other broadleaf weed species such as kochia (Kochia scoparia) and common waterhemp (Amaranthus rudis).

\subsection{Pre-Plant Control in Corn}

The GR giant ragweed was controlled 90 to $100 \%$ with all the evaluated pre-plant (soil applied) herbicides at 30 
DAT (Table 7). For example, the use of a photosystem II inhibitor (atrazine, $2240 \mathrm{~g}$ ai ha ${ }^{-1}$ ), HPPD inhibitor (isoxaflutole, $90 \mathrm{ai} \mathrm{ha}^{-1}$ ) or their tank-mixture provided 97 to $100 \%$ control of the GR giant ragweed 30 DAT. In addition, tank-mixture of atrazine (1120 $\mathrm{g}$ ai ha ${ }^{-1}$ ) plus a PPO inhibitor (flumioxazin, $210 \mathrm{~g}$ ai ha ${ }^{-1}$ ) or a premix of atrazine with a shoot growth inhibitor (dimethenamid-p, $723 \mathrm{~g}$ ai ha $^{-1}$ ) provided 93 to $100 \%$ control of the GR giant ragweed 30 DAT. All tested pre-plant herbicides provided 87 to $97 \%$ biomass reduction of the giant ragweed 30 DAT. Re-evaluation of the herbicide treatments at 60 or 75 DAT showed that high rate of saflufenacil (75 g ai $\left.\mathrm{ha}^{-1}\right)$ provided the lowest control $(57$ or $73 \%)$, followed by premix of flumioxazin/pyroxasulfone (85\%), while other herbicide treatments maintained 90 to $100 \%$ control of the giant ragweed. Similarly, in a study conducted at two locations near Windsor, Vink et al. (2012b) reported a tank-mix of saflufenacil and glyphosate provided $82 \%$ control of GR giant ragweed 30 DAT. In general, our study suggests that several herbicides with different modes of action are available for pre-plant control of GR giant ragweed.

Table 6. Effective doses of saflufenacil and dicamba at 21 DAT for $50 \%$ control or biomass reduction $\left(\mathrm{ED}_{50}\right)$ and $90 \%$ control or biomass reduction $\left(\mathrm{ED}_{90}\right)$ of 10,20 , or $30 \mathrm{~cm}$ tall giant ragweed

\begin{tabular}{|c|c|c|c|c|}
\hline \multirow[b]{2}{*}{ Weed height $(\mathrm{cm})$} & \multicolumn{2}{|c|}{ Control } & \multicolumn{2}{|c|}{ Biomass reduction } \\
\hline & $\mathrm{ED}_{50}( \pm \mathrm{SE})$ & $\mathrm{ED}_{90}( \pm \mathrm{SE})$ & $\mathrm{ED}_{50}( \pm \mathrm{SE})$ & $\mathrm{ED}_{90}( \pm \mathrm{SE})$ \\
\hline & \multicolumn{4}{|c|}{ 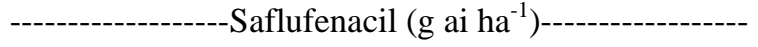 } \\
\hline 10 & $197(132)$ & $532(38)$ & $103(11)$ & $238(40)$ \\
\hline 20 & $227(108)$ & $495(101)$ & $210(79)$ & $437(233)$ \\
\hline \multirow[t]{2}{*}{30} & $652(373)$ & $810(221)$ & $265(87)$ & $804(280)$ \\
\hline & \multicolumn{4}{|c|}{-Dicamba $\left(g\right.$ ae ha $\left.{ }^{-1}\right)--$} \\
\hline 10 & $52(29)$ & $214(132)$ & $37(18)$ & $120(30)$ \\
\hline 20 & $210(40)$ & $402(102)$ & $146(18)$ & $252(15)$ \\
\hline 30 & $284(31)$ & $482(101)$ & $439(58)$ & $603(302)$ \\
\hline
\end{tabular}

Table 7. Pre-plant herbicide program on GR giant ragweed in Corn in 2013 and 2014

\begin{tabular}{|c|c|c|c|c|c|}
\hline \multirow[b]{2}{*}{ Herbicide } & \multirow[b]{2}{*}{ Rate } & \multicolumn{3}{|c|}{ Control } & \multirow{2}{*}{$\begin{array}{c}\text { Biomass reduction } \\
30 \\
\text { DAT }\end{array}$} \\
\hline & & $\begin{array}{c}30 \\
\text { DAT }\end{array}$ & $\begin{array}{c}60 \\
\text { DAT }\end{array}$ & $\begin{array}{c}75 \\
\text { DAT }\end{array}$ & \\
\hline & g ai ha ${ }^{-1}$ & $-\cdots$ & & $--\%$ & \\
\hline Atrazine & 2240 & 100 & $90 \mathrm{a}$ & $90 \mathrm{a}$ & 96 \\
\hline Isoxaflutole & 90 & 100 & $100 \mathrm{a}$ & $100 \mathrm{a}$ & 97 \\
\hline Isoxaflutole + Atrazine & $90+1120$ & 97 & $100 \mathrm{a}$ & $100 \mathrm{a}$ & 89 \\
\hline Mesotrione & 300 & 97 & $100 \mathrm{a}$ & $100 \mathrm{a}$ & 89 \\
\hline Thiencarbazone-methyl/isoxaflutole & 129 & 90 & $100 \mathrm{a}$ & $100 \mathrm{a}$ & 90 \\
\hline Flumioxazin/Pyroxasulfone & 315 & 100 & $85 \mathrm{~b}$ & $85 \mathrm{~b}$ & 91 \\
\hline Dimethenamid-P/atrazine & 723 & 100 & $100 \mathrm{a}$ & $100 \mathrm{a}$ & 96 \\
\hline S-metolachlor/mesotrione/atrazine & 2780 & 97 & $100 \mathrm{a}$ & $100 \mathrm{a}$ & 91 \\
\hline Saflufenacil & 75 & 90 & $73 \mathrm{c}$ & $57 \mathrm{c}$ & 87 \\
\hline Flumioxazin + Atrazine & $210+1120$ & 93 & $98 \mathrm{a}$ & $97 \mathrm{a}$ & 90 \\
\hline Saflufenacil/dimethenamid-P & 730 & 100 & $90 \mathrm{a}$ & $80 \mathrm{~b}$ & 94 \\
\hline $\begin{array}{l}\text { Mesotrione + S-metolachlor } \\
\operatorname{LSD}(\alpha \leq 0.05)\end{array}$ & 600 & 100 & $\begin{array}{c}100 \mathrm{a} \\
*\end{array}$ & $\begin{array}{c}100 \mathrm{a} \\
*\end{array}$ & 97 \\
\hline
\end{tabular}

${ }^{a}$ Abbreviations: DAT, days after treatment; data of non-treated plots were excluded in the analyses. Reduction in biomass was relative to biomass of non-treated plot.

${ }^{\mathrm{b}}$ Data within column with uncommon alphabet(s) are different based on Fisher's LSD test $(\alpha \leq 0.05)$.

${ }^{c}$ Herbicide premix (/); herbicide tank-mix (+ ); * Significant difference among treatments $(\alpha \leq 0.05)$.

\subsection{PRE followed by POST Control in Corn}

Most of the evaluated PRE herbicides provided 88 to $95 \%$ control of the GR giant ragweed, with an improved control up 100\% when PRE was followed by POST application of auxin herbicides (Table 8). For example, PRE application of atrazine (2240 $\mathrm{g}$ ai ha $\left.{ }^{-1}\right)$ or isoxaflutole $\left(90 \mathrm{ai} \mathrm{ha}^{-1}\right)$ provided 93 or $95 \%$ control $30 \mathrm{DAT}$, while a complete $(100 \%)$ control was recorded when followed by POST application of 2,4-D (535 $\mathrm{g}$ ae ha $\left.{ }^{-1}\right)$. The lowest 
(69\%) control provided PRE application of saflufenacil (75 $\left.\mathrm{g}_{\text {ai }} \mathrm{ha}^{-1}\right)$; however, POST application of dicamba $\left(66 \mathrm{~g}_{\text {ae }} \mathrm{ha}^{-1}\right.$ ) improved control to $100 \%$. Biomass was reduced at least $90 \%$ when PRE was followed by POST application of the any of the auxinic herbicides. Similarly, Vink et al. (2012b) reported at least $90 \%$ reduction in GR giant ragweed biomass with PRE followed by POST application of dicamba.

Table 8. PRE followed by (fb) POST herbicide program on GR giant ragweed in Corn in 2013 and 2014

\begin{tabular}{|c|c|c|c|c|}
\hline \multirow[b]{2}{*}{ Herbicide } & \multirow[b]{2}{*}{ Rate } & \multicolumn{2}{|c|}{ Control } & \multirow{2}{*}{$\begin{array}{c}\text { Biomass reduction } \\
30 \text { DAPOST }\end{array}$} \\
\hline & & 30 DAPRE & 30 DAPOST & \\
\hline & $\mathrm{g}$ ai $(\mathrm{ae}) \mathrm{ha}^{-1}$ & \multicolumn{3}{|c|}{ 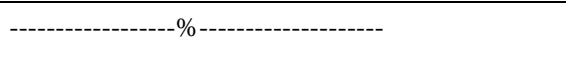 } \\
\hline Atrazine fb 2,4-D & $2240 \mathrm{fb} 535$ & $93 \mathrm{a}$ & 100 & 90 \\
\hline Isoxaflutole fb 2,4-D & $90 \mathrm{fb} 535$ & 94 a & 100 & 90 \\
\hline Isoxaflutole + atrazine fb 2,4-D & $90+1120$ fb 535 & 95 a & 100 & 95 \\
\hline Mesotrione fb flumetsulam/clopyralid & $300 \mathrm{fb} 54$ & $88 \mathrm{ab}$ & 100 & 94 \\
\hline Thiencarbazone-methyl/isoxaflutole fb 2,4-D & $129 \mathrm{fb} 535$ & 95 a & 100 & 95 \\
\hline 2,4 D (POST-only) & 535 & $10 \mathrm{~d}$ & 91 & 90 \\
\hline Dimethenamid-P/atrazine fb 2,4-D & $723 \mathrm{fb} 535$ & 93 a & 100 & 94 \\
\hline S-metolachlor/mesotrione/atrazine fb 2,4-D & $2780 \mathrm{fb} 535$ & $94 \mathrm{a}$ & 100 & 93 \\
\hline Saflufenacil fb diflufenzopyr/dicamba & $75 \mathrm{fb} 360$ & $69 c$ & 100 & 96 \\
\hline Saflufenacil/dimethenamid-P fb diflufenzopyr/dicamba & $730 \mathrm{fb} 360$ & $87 \mathrm{~b}$ & 100 & 96 \\
\hline Mesotrione + S-metolachlor fb diflufenzopyr/dicamba & $600 \mathrm{fb} 360$ & $89 \mathrm{ab}$ & 100 & 95 \\
\hline $\operatorname{LSD}(\alpha \leq 0.05)$ & & $*$ & & \\
\hline
\end{tabular}

${ }^{a}$ Abbreviations: DAPRE, days after PRE; DAPOST, days after POST; data of non-treated plots were excluded in the analyses. Reduction in biomass was relative to biomass of non-treated plot.

${ }^{\mathrm{b}}$ Data within column with uncommon alphabet(s) are different based on Fisher's LSD test $(\alpha \leq 0.05)$.

${ }^{\mathrm{c}}$ Herbicide premix (/); herbicide tank-mix (+ )

*Significant difference among treatments $(\alpha \leq 0.05)$.

Table 9. PRE followed by (fb) POST herbicide program on GR giant ragweed in Soybean in 2013 and 2014

\begin{tabular}{|c|c|c|c|c|}
\hline \multirow[b]{2}{*}{ Herbicide } & \multirow[b]{2}{*}{ Rate } & \multicolumn{2}{|c|}{ Control } & \multirow{2}{*}{$\frac{\text { Biomass reduction }}{\text { 30DAPOST }}$} \\
\hline & & 30 DAPRE & 30 DAPOST & \\
\hline & g ai ha ${ }^{-1}$ & ------------- & -.. & \\
\hline Sulfentrazone/cloransulam fb imazamox + acifluorfen & $392 \mathrm{fb} 35+280$ & $100 \mathrm{a}$ & 100 & 98 \\
\hline $\begin{array}{l}\text { Chlorimuron/flumioxazin/thifensulfuron fb } \\
\text { imazamox + acifluorfen }\end{array}$ & $106 \mathrm{fb} 35+280$ & $100 \mathrm{a}$ & 100 & 99 \\
\hline Clorasulam fb lactofen & $10 \mathrm{fb} 219$ & $100 \mathrm{a}$ & 100 & 97 \\
\hline $\begin{array}{l}\text { Flumioxazin/clorasulam + flumioxazin + } \\
\text { clorasulam fb imazamox + acifluorfen }\end{array}$ & $129+75+10 \mathrm{fb} 35+280$ & $100 \mathrm{a}$ & 100 & 96 \\
\hline Saflufenacil/imazethapyr fb lactofen & $100 \mathrm{fb} 219$ & $90 \mathrm{ab}$ & 100 & 98 \\
\hline Imazethapyr fb lactofen & $70 \mathrm{fb} 219$ & $90 \mathrm{ab}$ & 98 & 96 \\
\hline Imazethapyr + S-metolachlor fb lactofen & $70+1411 \mathrm{fb} 219$ & $85 \mathrm{~b}$ & 100 & 96 \\
\hline Imazaquin fb lactofen & $143+1411 \mathrm{fb} 219$ & $100 \mathrm{a}$ & 100 & 96 \\
\hline Metribuzin fb lactofen & $438 \mathrm{fb} 219$ & $84 \mathrm{~b}$ & 98 & 94 \\
\hline Flumioxazin fb clorasulam & $113 \mathrm{fb} 5$ & $98 \mathrm{a}$ & 100 & 96 \\
\hline $\begin{array}{l}\text { Flumioxazin/Chlorimuron fb imazamox }+ \text { acifluorfen } \\
L S D(\alpha \leq 0.05)\end{array}$ & $158 \mathrm{fb} 35+280$ & $\begin{array}{c}100 \mathrm{a} \\
*\end{array}$ & 100 & 98 \\
\hline $\begin{array}{l}\text { Abbreviations: DAPRE, days after PRE; DAPOST, day } \\
\text { iomass was relative to biomass of non-treated plot. }\end{array}$ & fter POST; data of non-tre & ed plots were & excluded in the & nalyses. Reduction \\
\hline
\end{tabular}


Table 10. POST-only herbicide program on GR giant ragweed in Soybean in 2013 and 2014

\begin{tabular}{lccc}
\hline Herbicide & Rate & $\begin{array}{c}\text { Control } \\
30 \text { DAT }\end{array}$ & $\begin{array}{c}\text { Biomass reduction } \\
\text { 30 DAT }\end{array}$ \\
\hline & $\mathrm{g} \mathrm{ai} \mathrm{ha}^{-1}$ & $---------\%$ & --------- \\
Clorasulam & 5 & $80 \mathrm{~b}$ & $75 \mathrm{~b}$ \\
Thifensulfuron + chlorimuron & $5+5$ & $40 \mathrm{c}$ & $41 \mathrm{c}$ \\
Lactofen & 219 & $85 \mathrm{~b}$ & $82 \mathrm{~b}$ \\
Imazamox + acifluorfen & $35+280$ & $62 \mathrm{c}$ & $61 \mathrm{c}$ \\
Fomesafen/glyphosate & 1382 & $96 \mathrm{a}$ & $92 \mathrm{a}$ \\
$L S D(\alpha \leq 0.05)$ & & $*$ & $*$ \\
\hline
\end{tabular}

\footnotetext{
${ }^{a}$ Abbreviations: DAT, days after treatment; data of non-treated plots were excluded in the analyses. Reduction in biomass was relative to biomass of non-treated plot.

${ }^{b}$ Data within column with uncommon alphabet(s) are different based on Fisher's LSD test $(\alpha \leq 0.05)$

${ }^{\mathrm{c}}$ Herbicide premix (/); herbicide tank-mix (+)

*Significant difference among treatments $(\alpha \leq 0.05)$.
}

\subsection{PRE followed by POST Control in Soybean}

The GR giant ragweed population was controlled 90 to $100 \%$ with most of the evaluated PRE herbicides, with a sustained or improved control with POST application of herbicides (Table 9). For example, PRE applied premix of sulfentrazone/cloransulam (392 $\mathrm{g}$ ai $\mathrm{ha}^{-1}$ ) provided 100\% control of giant ragweed 30 days after PRE (DAPRE); this level of control was sustained with POST applied tank-mix of imazamox (35 $\mathrm{g}$ ai ha ${ }^{-1}$ ) plus acifluorfen (280 $\left.\mathrm{g}_{\text {ai }} \mathrm{ha}^{-1}\right) 30$ days after POST (DAPOST). In addition, PRE application of imazethapyr (70 $\mathrm{g}$ ai $\mathrm{ha}^{-1}$ ) provided $90 \%$ control, while $100 \%$ control was achieved when followed by POST application of lactofen $\left(219 \mathrm{~g}\right.$ ai ha $\left.^{-1}\right) 30$ DAPOST.

The least control (84\%) was provided by PRE application of metribuzin (438 $\mathrm{g}$ ai ha $\left.\mathrm{a}^{-1}\right) 30$ DAPOST; however, 98\% control was achieved when followed by POST application of lactofen $\left(219 \mathrm{~g}\right.$ ai ha $\left.{ }^{-1}\right)$. Giant ragweed biomass reduction with the herbicide programs was similar to the visual control ratings 30 DAPOST (Table 8). These results indicates that there are available herbicide options for GR giant ragweed control in soybean.

\subsection{POST-only Control in Soybean}

POST-only herbicide program provided $\leq 90 \%$ GR giant ragweed control, except POST-application of fomesafen/glyphosate (1380 g ai ha ${ }^{-1}$ ) which provided $96 \%$ control 30 DAT (Table 10). The poor control of giant ragweed by the POST-only herbicides may be attributed to high ragweed density and taller plants at the time of application. Giant ragweed usually emerges early in the growing season; thus, a delayed POST weed control program would result in poor giant ragweed control. Previous research has shown that without pre-plant or PRE weed control, POST-only herbicide program could provide undesirable control of GR giant ragweed (Ganie et al., 2016; Follings et al., 2013).

Weed control programs based on rotation of herbicides or mixtures of multiple site of actions has been widely recommended to minimize selection pressure often associated with evolution of resistant weeds (Knezevic et al., 2009; Robinson et al., 2012; Regnier et al., 2016; Osipitan and Dille, 2017; Evans et al., 2018). In addition, risk of herbicide resistance may be reduced when PRE followed by POST application programs are used as part of diverse approach to weed control (Livingston et al. 2015). Research also showed that that sequential application of PRE followed by POST herbicides would control cohorts that emerge over longer period of time in the growing season, especially early- and mid-season emerging weeds (Vink et al., 2012a; Kaur et al., 2016). In general, it is recommended that PRE followed by POST herbicide program would be a good tool for GR giant ragweed control in corn and soybean. Results from this study confirmed that giant ragweed population near David city, Nebraska was truly glyphosate resistant and that dicamba can effectively control the GR giant ragweed, particularly when applied at early growth stage (10 to $20 \mathrm{~cm}$ tall) of the weed. Most importantly and from the practical standpoint, we determined that there are herbicide programs available to effectively control GR ragweed in corn and soybean, at least under Nebraska's growing conditions. 


\section{References}

Belfry, K. D., \& Sikkema, P. H. (2015). Preplant and postemergence control of glyphosate-resistant giant ragweed in corn. Agric Sci., 6, 250-256. https:// doi.org/10.4236/as.2015.62026

Chahal, P. S., Aulakh, J. S., Rosenbaum, K., \& Jhala, A. J. (2015). Growth stage affects dose response of selected glyphosate-resistant weeds to premix of 2, 4-D choline and glyphosate (Enlist Duo ${ }^{\mathrm{TM}}$ Herbicide* $^{*}$. J Agric Sci., 7, 1-9. https:// doi.org/10.5539/jas.v7n11p1

Ditschun, S., Soltani, N., Robinson, D. E., Tardif, F. J., Kaastra, A. C., \& Sikkema, P. H. (2016). Control of Glyphosate-Resistant Giant Ragweed (Ambrosia trifida L.) with Isoxaflutole and Metribuzin Tankmix. Am J Plant Sci., 7, 900-916. https://doi.org/10.4236/ajps.2016.76087

Evans, J. A., Williams, A., Hager, A. G., Mirsky, S. B., Tranel, P. J., \& Davis, A. S. (2018). Confronting herbicide resistance with cooperative management. Pest Manag Sci., 8, 123. https://doi.org/0.1002/ps.5105

Follings, J., Soltani, N., Robinson, D. E., Tardif, F. J., Lawton, M. B., \& Sikkema, P. H. (2013). Glyphosate-resistant giant ragweed. Agric Sci., 4, 194. https://doi.org/10.4236/as.2013.44028

Ganie, Z. A., Sandell, L. D., Jugulam, M., Kruger, G. R., Marx, D. B., \& Jhala, A. J. (2016). Integrated management of glyphosate-resistant giant ragweed (Ambrosia trifida) with tillage and herbicides in soybean. Weed Technol., 30, 45-56. https://doi.org/10.1614/WT-D-15-00089.1

Goplen, J. J., Sheaffer, C. C., Becker, R. L., Coulter, J. A., Breitenbach, F. R., Behnken, L. M., ... Gunsolus, J. L. (2017). Seedbank Depletion and Emergence Patterns of Giant Ragweed (Ambrosia trifida) in Minnesota Cropping Systems. Weed Sc., 65, 52-60. https://doi.org/10.1614/WS-D-16-00084.1

Goplen, J. J., Coulter, J. A., Sheaffer, C. C., Becker, R. L., Breitenbach, F. R., Behnken, L. M., \& Gunsolus, J. L. (2018). Economic Performance of Crop Rotations in the Presence of Herbicide-Resistant Giant Ragweed. Agron J., 110, 260-268. https://doi.org/10.2134/agronj2016.09.0536

Harrison, S. K., Regnier, E. E., Schmoll, J. T., \& Webb, J. E. (2001). Competition and fecundity of giant ragweed in corn. Weed Sci., 49(2), 224-229. https://doi.org/10.1614/0043-1745

Heap, I. (2018). The International Survey of Herbicide Resistant Weeds. http://www.weedscience.com. Accessed: August 10, 2018

Heap, I., \& Duke, S. O. (2018). Overview of glyphosate - resistant weeds worldwide. Pest Manag Sci., 74, 1040-1049. https://doi.org/10.1002/ps.4760

Kaur, S., Sandell, L. D., Lindquist, J. L., \& Jhala, A. J. (2014). Glyphosate-resistant giant ragweed (Ambrosia trifida) control in glufosinate-resistant soybean. Weed Technol., 28, 569-577. https://doi.org/10.1614/WT-D-14-00009.1

Kaur, S., Werle, R., Sandell, L., \& Jhala, A. J. (2016). Spring-tillage has no effect on the emergence pattern of glyphosate-resistant giant ragweed (Ambrosia trifida L.) in Nebraska. Can J Plant Sci., 96, 726-729. https://doi.org/10.1139/cjps-2015-0287

Knezevic, S. Z., Streibig, J. C., \& Ritz, C. (2007). Utilizing R software package for dose-response studies: the concept and data analysis. Weed Technol., 21, 840-848. https://doi.org/10.1614/WT-06-161.1

Knezevic, S. Z., Datta, A., Scott, J., Klein, R. N., \& Golus, J. (2009). Problem weed control in glyphosate-resistant soybean with glyphosate tank mixes and soil-applied herbicides. Weed Technol., 23, 507-512. https://doi.org/10.1614/WT-09-012.1

Knezevic, S. Z., Osipitan, O. A., \& Scott, J. E. (2018). Sensitivity of Grape and Tomato to Micro-rates of Dicamba-based Herbicides. J Hortic., 5, 1.

Koger, C. H., Poston, D. H., Hayes, R. M., \& Montgomery, R. F. (2004). Glyphosate-resistant horseweed (Conyza canadensis) in Mississippi. Weed Technol., 18, 820-825. https://doi.org/10.1614/WT-03-218R

Livingston, M., Fernandez-Cornejo, J., Unger, J., Osteen, C., Schimmelpfennig, D., Park, T., \& Lambert, D. (2015). The Economics of Glyphosate Resistance Management in Corn and Soybean Production, Washington, DC: US Department of Agriculture ERR. 184, 45.

Mahoney, K. J., McNaughton, K. E., \& Sikkema, P. H. (2015). Control of glyphosate-resistant giant ragweed in winter wheat. Weed Technol., 29, 868-873. https://doi.org/10.1614/WT-D-15-00028.1

Norsworthy, J. K., Jha, P., Steckel, L. E., \& Scott, R. C. (2010). Confirmation and control of glyphosate-resistant giant ragweed (Ambrosia trifida) in Tennessee. Weed Technol., 24, 64-70. 
https://doi.org/10.1614/WT-D-09-00019.1

Osipitan, O. A., \& Dille, J. A. (2017). Fitness outcomes related to glyphosate resistance in kochia (Kochia scoparia): what life history stage to examine?. Front. Plant Sci., 8, 1090. https://doi.org/10.3389/fpls.2017.01090

Osipitan, O. A., Scott, J. E., \& Knezevic, S. Z. (2018). Tolpyralate applied alone and with atrazine for weed control in corn. J Agric Sci., 10, 32-39. https://doi.org/10.5539/jas.v10n10p32

R Development Core Team (2018). R: A Language and Environment for Statistical Computing. R Foundation for Statistical Computing, Vienna, Austria. Retrieved from http://www.R-project.org.

Regnier, E. E., Harrison, S. K., Loux, M. M., Holloman, C., Venkatesh, R., Diekmann, F., \& Davis, A. S. (2016). Certified crop advisors' perceptions of giant ragweed (Ambrosia trifida) distribution, herbicide resistance, and management in the Corn Belt. Weed Sci., 64, 361-377. https://doi.org/10.1614/WS-D-15-00116.1

Riley, E. B., \& Bradley, K. W. (2014). Influence of application timing and glyphosate tank-mix combinations on the survival of glyphosate-resistant giant ragweed (Ambrosia trifida) in soybean. Weed Technol., 28, 1-9. https://doi.org/10.1614/WT-D-13-00098.1

Robinson, A. P., Simpson, D. M., \& Johnson, W. G. (2012). Summer annual weed control with 2,4-D and glyphosate. Weed Technol., 26, 657-660. https://doi.org/10.1614/WT-D-12-00081.1

Schrage, B. W. (2018). Confirmation and Control of Glyphosate-, ALS-, and PPO-Resistant Common Ragweed (Ambrosia artemisiifolia) in North Carolina. PhD thesis, North Carolina State University. 76-102.

Shrestha, A., Hembree, K., \& Va, N. (2007). Growth stage influences level of resistance in glyphosate-resistant horseweed. California Agric., 61, 67-70. https://doi.org/10.3733/ca.v061n02p67

Spaunhorst, D. J., Siefert-Higgins, S., \& Bradley, K. W. (2014). Glyphosate-resistant giant ragweed (Ambrosia trifida) and waterhemp (Amaranthus rudis) management in dicamba-resistant soybean (Glycine max). Weed Technol., 28, 131-141. https://doi.org/WT-D-13-00091.1

VanGessel, M. (2001). Glyphosate-resistant horseweed from Delaware. Weed Sci., 49,703-705. https://doi.org/10.1614/0043-1745

Vink, J. P., Soltani, N., Robinson, D. E., Tardif, F. J., Lawton, M. B., \& Sikkema, P. H. (2012a). Glyphosate-resistant giant ragweed (Ambrosia trifida) control in dicamba-tolerant soybean. Weed Technol., 26, 422-428. https://doi.org/10.1614/WT-D-11-00184.1

Vink, J. P., Soltani, N., Robinson, D. E., Tardif, F. J., Lawton, M. B., \& Sikkema, P. H. (2012b). Glyphosateresistant giant ragweed (Ambrosia trifida L.) control with preplant herbicides in soybean [Glycine max (L.) Merr.]. Can J of Plant Sci., 92, 913-922. https://doi.org/10.4141/cjps2012-025

Wuerffel, R. J., Young, J. M., Matthews, J. L., Davis, V. M., Johnson, W. G., \& Young, B. G. (2015). Timing of soil-residual herbicide applications for control of giant ragweed (Ambrosia trifida). Weed Technol., 29, 771-781. https://doi.org/10.1614/WT-D-15-00018.1

\section{Copyrights}

Copyright for this article is retained by the author(s), with first publication rights granted to the journal.

This is an open-access article distributed under the terms and conditions of the Creative Commons Attribution license (http://creativecommons.org/licenses/by/3.0/). 